\title{
Concepciones populares sobre soledad de los adultos mayores de España y Bucaramanga, Colombia
}

\section{Popular concepts on loneliness of the elderly people of Spain and Bucaramanga, Colombia}

\author{
Ramona Rubio Herrera \\ Gabinete de Calidad de Vida y \\ Envejecimiento, Universidad de Granada, \\ Granada, España \\ Ara Mercedes Cerquera Córdoba \\ Universidad Pontificia Bolivariana \\ Seccional Bucaramanga, Colombia \\ Rubiela Muñoz Mejía \\ Universidad Pontificia Bolivariana \\ Seccional Bucaramanga, Colombia

\section{Erika Alejandra Pinzón Benavides \\ Gabinete de Calidad de Vida y \\ Envejecimiento, Universidad Pontificia \\ Bolivariana Seccional Bucaramanga, \\ Colombia} \\ Universidad de Granada, Granada, España \\ Recibido: 25 de febrero de 2011 \\ Revisado: 31 de mayo de 2011 \\ Aceptado: 12 de julio de 2011
}

\section{Resumen}

El propósito del presente artículo es comparar las concepciones populares de soledad de los adultos mayores de España y los adultos mayores de Bucaramanga, Colombia; para ello se utilizó un diseño cualitativo comparativo de los resultados arrojados por la "Encuesta Concepción de Soledad" con el ítem número 16 del cuestionario "La soledad en las personas mayores". La muestra fue conformada por 1956 adultos mayores de España y 6607 adultos mayores de Bucaramanga, Colombia. Los criterios de inclusión tomados en cuenta en las 2 poblaciones fueron ser mayor de 65 años. Entre los resultados se encuentra predominancia de las concepciones negativas sobre la soledad, alta incidencia en concepciones relacionadas con la familia, categorías que hacen referencia a la soledad objetiva y subjetiva, concepciones positivas presentes en la población colombiana.

Palabras clave: soledad, adultos mayores, vejez, envejecimiento, ciclo vital, Calidad de vida.

Artículo de investigación. Trabajo de investigación realizado a partir del proceso de Práctica de Psicología de la Universidad Pontificia Bolivariana, Seccional Bucaramanga, en convenio con la Universidad de Granada, España. La práctica se llevó a cabo en el Gabinete de Calidad de Vida y Envejecimiento de la Universidad de Granada, España durante el periodo comprendido entre octubre 2010 y febrero de 2011.

Correspondencia: Ramona Rubio Herrera. Directora del Gabinete de Calidad de Vida y Envejecimiento, Universidad de Granada, Granada, España. Correo electrónico: rrubio@ugr.es. Ara Mercedes Cerquera Córdoba. Universidad Pontificia Bolivariana, Seccional Bucaramanga, Colombia. Correo electrónico: ara.cerquera@upb.edu.co. Rubiela Muñoz Mejía. Universidad Pontificia Bolivariana, Seccional Bucaramanga, Colombia. Correo electrónico: rubiela.muñoz@upb.edu.co. Alejandra Pinzón Benavides. Universidad Pontificia Bolivariana, Seccional Bucaramanga, Colombia. Universidad de Granada, Granada, España. Correo electrónico: erika.pinzon@upb.edu.co, rika_alejandrap@hotmail.com 


\section{Abstract}

The goal of the followingarticleis to compare the concepts of loneliness in the elderly people of Spain and the elderly people of Bucaramanga, Colombia; this involved the use of a comparative qualitative design of the results of the "Conception of Loneliness Survey" against item 16 of the questionnaire "The loneliness of the elderly". The sample consisted of 1956 Spanish elders and 6607 of Bucaramanga, Colombia. A minimum age of 65 was held as a constant between both samples. The results showed a predominant negative conception of loneliness, high incidence of family related concepts, categories referencing isolation (objective solitude) and loneliness (subjective solitude), and positive concepts in the Colombian sample.

Keywords: loneliness, elderly people, oldness, aging, life span, quality of life.

La soledad es un tema que compete a los seres humanos en general, en algún momento de la vida cualquier persona sin importar su edad, sexo o estrato social ha experimentado soledad, pero al mismo tiempo la soledad suele ser un tema debate, un constructo que ha sido difícil de definir, porque comprende un sin número de concepciones humanas que dependen de la época, cultura, historia de vida y estilo de vida que lleva cada ser humano, desde esta perspectiva, como indican Rubio, Rubio y Pinel (2009) la soledad constituye un macroconcepto que engloba muchos y diferentes tipos de definiciones.

Sin embargo, a pesar de que existen diversos fenómenos u objetos de estudio en relación con los adultos mayores, la soledad no deja de ser un tema de interés en este momento de la vida, pues la sociedad se ha encargado de asociarla con una visión negativa de la vejez.

Teniendo en cuenta la teoría del ciclo vital propuesta por Baltes (1987), el envejecimiento es un proceso natural del ser humano, todos envejecemos, desde el mismo momento en que empezamos a vivir. La vejez, entonces, sería una parte de ese ciclo vital, un momento de ese proceso. Para dar mayor claridad a la diferenciación entre estos dos conceptos según Parales y Dulcey (2002) la vejez se relaciona con realidades más evidentes e identificables en el presente, el concepto de envejecimiento se refiere más a un proceso, a una diacronía mucho menos manifiesta y precisa.

Pero, estos dos términos han sido degradados.
Piña (2004) indica que la vejez, muchas veces, trae consigo una serie de mitos y estigmas que han sido impuestos no solo por la sociedad en la que se desenvuelve el adulto mayor si no por ellos mismos, considerando que la vejez es un periodo de perdidas relacionado con la muerte y la decadencia, lo anterior resume lo que Salvarezza (2002) llama el fenómeno del viejísimo.

Comúnmente las personas en general, de todas las edades, inclusive los mismos mayores, perciben la vejez desde una posición negativa; se puede decir que temen llegar a la vejez o envejecer y desconocen que el envejecimiento no es exclusivo de este periodo de vida, sino que es inherente al ser humano y a todos los momentos del ciclo vital, de esta manera se puede evidenciar que la vejez se ha degradado desde muchas facetas y expresiones humanas, empezando por los medios de comunicación que diariamente la relacionan con la enfermedad, la muerte, el deterioro, la fealdad, la debilidad, entre otros, relaciones que desarrollan actitudes, creencias y acciones que estimulan la predispocición, el rechazo y la negación a este periodo vital.

Según Baltes (1987) el desarrollo humano se entiende como un proceso que se da a lo largo de la vida, que involucra ganancias y pérdidas, es multidimensional, multicausal, implica aspectos tanto biológicos como culturales, es modificable e influido por el contexto; además, afirma que la vejez es una etapa del ciclo vital, que como cualquier otra conlleva a una serie de transformaciones y cambios en el individuo; ésta se ha venido enmarcando bajo una concepción degenerativa, 
producto de definiciones brindadas por la biología y la ciencia de la salud, las cuales se centran en el deterioro generado por la disminución de células que se forman en el organismo.

Estas definiciones se basan solo en la esfera biológica y olvidan incluir una parte muy importante, pues la vejez también encierra una serie de variaciones que se presentan no solo a nivel físico sino además a nivel familiar, personal, laboral y social, que tienen en cuenta no solo las pérdidas sino también las ganancias, como cualquier periodo del ciclo vital.

Por otra parte, un aspecto importante para reflexionar es

el envejecimiento es una realidad del ser humano que debe considerarse a nivel individual, pero también un fenómeno colectivo, ya que no solo aumenta el número de personas mayores cada día, si no que se ha incrementado la esperanza de vida, produciéndose un envejecimiento interno de la población (Botella, 2005, en Pinazo \& Sánchez, 2005, p. 93).

El envejecimiento poblacional es un cambio que se debe a diversos factores de tipo histórico, cultural, demográfico, político-social, biológico, entre otros, pero sobre todo a la transición demográfica o los descensos en las tasas de fecundidad y mortalidad. La población mayor se está aumentado cada día y no solo involucra a los adultos mayores, sino también a la sociedad como tal; esta se debe preparar para atender las necesidades de las personas mayores y al mismo tiempo para afrontar cambios a nivel social y económico.

La historia evidencia claramente como se ha venido presentando este acontecimiento social: "para la época del Imperio Romano el promedio de vida oscilaba alrededor de los 23 años de edad, para la época del descubrimiento de América era alrededor de 30 años, en la era victoriana, el promedio de vida alcanzaba los 45 años y actualmente rebasa los 75 años” (Rodríguez, 2010, p. 93).

Se suele pensar que este fenómeno es exclusivo de países desarrollados, argumentando la idea de que es visible en ellos, pero no es así; aunque se ha manifestado más aceleradamente en los países desarrollados, no implica su ausencia en países en vía de desarrollo, actualmente es un fenómeno que comprende a la población mundial, un ejemplo de ello se puede evidenciar en las siguientes estadísticas.

Según López (2004) el Censo de 2001 otorgaba a España casi 41 millones de habitantes, siete millones de ellos (más de un 17\%) con más de 65 años, presentándose como una magnitud máxima histórica, puesto que nunca se había contado en España con tantas personas mayores. "De sobra es conocido que la población española se encuentra experimentando de lleno un proceso de envejecimiento, proceso que empezó a manifestarse en fechas tardías, pero que ha acelerado su ritmo enormemente durante las últimas décadas transcurridas" (López, 2004, p. 20).

De la misma manera las estadísticas arrojadas por el Departamento Administrativo Nacional de Estadísticas - DANE (2005) evidencian el aumento progresivo de la población mayor colombiana; en 1975 el 5,6\% correspondía a personas mayores de 60 años, en el 2005 al 9\%, y se estima que en el 2025 aumente al 13,5\%, esto quiere decir que hacia el año 2050 el total de la población colombiana oscilará entre 72 millones de habitantes, y la esperanza de vida será superior a los 79 años, con más del $20 \%$ de los pobladores por encima de 60 años. "El envejecimiento de la población colombiana se viene dando desde mediados del siglo XX, como consecuencia del proceso de transición" (Zamudio et ál., 2003, p. 5).

A pesar de un acelerado crecimiento de la población mayor y del aumento de la esperanza de vida, surgen diferencias sociales entre los contextos mencionados, diferencias que deben ser analizadas desde los fenómenos que se presentan en relación con la población mayor.

Guzmán y Huenchuan en compañía del Centro Latinoamericano y Caribeño de Demografía [CELADE-CEPAL] (2006) exponen los resultados de un estudio de la seguridad de ingresos en la vejez. Entre los resultados arrojados en la población 
colombiana del 2002 se encuentran porcentajes alarmantes como un $73 \%$ de mayores sin ingresos, un $6 \%$ con ingresos de su trabajo, un $21 \%$ con ingresos del trabajo y la jubilación y un nivel de pobreza de la población mayor del $40 \%$.

Lo anterior justifica la importancia de investigar sobre los fenómenos que aquejan a la población mayor en diferentes partes del mundo, como por ejemplo la soledad, ya que se desconoce cómo los adultos mayores la vivencian y perciben, los efectos generados, factores influyentes, características de afrontamiento, entre otros.

Cada día aumentan más las cifras de mayores que viven solos, que son institucionalizados o que presentan síntomas asociados con la depresión, pero son escasas las investigaciones que incluyen la opinión y la concepción de la soledad por los mismos mayores.

Por tanto, no es igual juzgar sobre la soledad de los mayores sin tomarse a la tarea de preguntarles a ellos mismos qué es la soledad. "La soledad no es un patrimonio de generaciones pasadas ni presentes. La soledad es un problema real de todos los cohortes y generaciones" (Rubio, 2004, p. 4).

No se puede atribuir a la soledad una sola población, una época en particular o un espacio en específico, la soledad es un constructo que compromete a muchas poblaciones, épocas y espacios, es incluso un término utilizado a diario por cualquier tipo de ciudadano. Soledad, se ve en las calles, en los espacios cerrados, en los estratos altos y bajos, en las instituciones, en la población juvenil y por supuesto también en el adulto mayor.

Muchas veces se mantiene el estereotipo de que la soledad va ligada a la vejez, si bien es cierto que muchos de los adultos mayores experimentan soledad por diversos factores asociados o no a este momento de vida, esto no indica que los mayores sean los únicos que la experimenten.

Como lo indica el Boletín sobre envejecimiento del Ministerio de Trabajo y Asuntos Sociales en España [MTAS] (2000) la llegada a la vejez implica una serie de cambios vitales que van más allá del aumento de años. Adaptarse a esta nueva vida no es tarea fácil y depende de los recursos personales, familiares y sociales de las personas mayores. A causa de estos cambios pueden experimentar soledad.

Diversos autores han estudiado la influencia que ejercen estos cambios vitales sobre la soledad y así mismo la calidad de vida, Iglesias (2001) indagó las influencias personales, familiares y sociales, encontrando relación entre la soledad, jubilación y viudez. Esta investigación indica que la soledad puede estar asociada con los cambios y aspectos que se dan en la vejez como periodo de vida.

Rojo (2010) investigó la calidad de vida de las personas mayores, encontrando que la salud, la red familiar, las redes sociales, las actividades de ocio, se presentan como factores determinantes de la percepción de los mayores sobre su calidad de vida.

Rubio (2007) define la soledad como un malestar emocional que se genera cuando un individuo se siente incomprendido y rechazado por otro o cuando éste carece de compañía para la realización de aquellas actividades que desea, sean éstas, físicas, intelectuales o emocionales.

Estas definiciones sobre soledad se han enmarcado desde una concepción negativa de la misma, mostrándola como una experiencia indeseable.

Según Rubio (2009) existen diferentes estudios de la soledad que van desde abordar la soledad objetiva o "isolation", sus consecuencias psíquicas (Seligman, 1975), como consecuencia de la vida interna en instituciones (Curry \& Ratliff, 1973; Levine, 1980, Silverstone \& Miller, 1980), desde el abandono o de la enfermedad (Managan, 1974, Evans, Werkhoven \& Fox, 1982), como un mecanismo de defensa (Myerson, 1980), entre muchos más, y otros que lo hacen desde la soledad subjetiva o "loneliness".

Según Rubio (2004) las concepciones de soledad se puede ver desde dos dimensiones: la soledad objetiva que indica el vivir solo y la soledad subjetiva que supone el sentirse solo. 
Estas concepciones suponen que existen diferentes formas de definir y experimentar soledad y que se podrían considerar diferentes tipos de soledad, de la misma manera confrontan la idea de que la soledad se relaciona solo con las ausencias.

En su faceta más negativa, la soledad constituye uno de los principales problemas que afectan cotidianamente aquellos mayores que habitan en hogares unipersonales. Este mal es interpretado como consustancial al tipo de vida que llevan a cabo y puede adquirir un doble sentido o dimensión (López, 2004, p. 188).

\section{Acercamiento a los estudios sobre soledad}

Dentro de las investigaciones sobre soledad en adultos mayores cabe resaltar al Instituto de Migraciones Sociales [IMSERSO] y Centro de Investigaciones Sociológicas [CIS] en Coordinación de Rubio (1999), quienes realizaron un estudio de la soledad con 2500 mayores españoles; este incluía diversos aspectos como datos sociodemográficos, datos familiares, hábitos y conceptos populares. Entre uno de los resultados se encontró que la definición popular de la soledad era asociada en mayor escala a aspectos negativos como sentimiento de vacío y tristeza, pérdida de personas queridas y falta de contactos sociales, es decir, nostalgias derivadas de ausencias.

Rubio y Aleixandre (1999) diseñaron la Escala de Soledad [ESTE] a partir de las Escalas UCLA, la ESLI, la SELSA y Satisfacción Vital de Philadelphia. Esta escala de soledad cuenta con 4 factores: soledad familiar, conyugal, social y existencial.

En el 2001, Rubio y Aleixandre estudiaron las concepciones y autopercepción de la soledad de los mayores españoles en cuanto a estar solo y sentirse solo, encontrando que a pesar de presentarse teorías implícitas de la soledad como un sentimiento subjetivo, los sujetos que viven solos generan más índices de soledad que los que viven acompañados, es decir, que el $74 \%$ de los que viven solos perciben la soledad.
Rubio (2004) a través de la Escala ESTE, midió la soledad en personas mayores españolas, de la ciudad de Granada, relacionada con la edad, el género, la escolaridad y el estado civil, encontrando un incremento de la soledad percibida a los 94 años, puntuaciones más elevadas en sujetos sin pareja e índices de soledad superiores en mujeres; de la misma manera la escala permitió analizar la soledad desde los siguientes factores familiar, conyugal, social y existencial.

En el 2009, Rubio abordó la soledad en la población del adulto mayor español, en relación con la percepción del apoyo, el índice de participación subjetiva y el uso de nuevas tecnologías. De esta manera define la soledad como "la experiencia subjetiva de insatisfacción frente al distanciamiento respecto a la sociedad en la que vive el adulto mayor, ocasionando la pérdida de ritmo en los cambios sociales de la nueva era digital y provocando la falta de adaptación a las nuevas tecnologías" (Rubio, 2009, p. 3).

En diversos estudios (Rubio, Rubio \& Pinel, 2009a, 2009b) indagaron la soledad de las personas mayores españolas relacionada con variables sociodemográficas y experiencia subjetiva de insatisfacción frente a la sociedad actual, a través de la revisión de la Escala ESTE [ESTE- R] y la creación de la Escala de soledad social [ESTE- II].

Cardona, Henao, Quintero y Villamil (2009) exploraron el concepto de soledad de los adultos mayores no institucionalizados de Bello, Colombia, encontrando que predomina una concepción más objetiva (estar solo) que subjetiva (sentirse solo) de soledad y una estrecha relación entre la manera como se percibe el momento actual de vida con el concepto de soledad.

En el 2010, la Línea de Calidad de Vida en el Adulto Mayor de la Universidad Pontificia Bolivariana, realizó un estudio de las concepciones populares de soledad de los adultos mayores de Bucaramanga, Colombia, utilizando una muestra de 6607 personas mayores; entre los resultados encontraron una mayor frecuencia en las categorías de "estar solo", "sentirse solo", "tristeza”, 
y categorías que indican una percepción positiva como "bienestar".

Por otra parte, en diversas investigaciones (Alfageme, 1999; Arranz, Giménez, De Castro, Baeza \& De la Fuente, 2009; Bermejo, 2005; Bondevik \& Skogstad, 1996; Del Barrio et ál., 2010; Pettigrew \& Roberts, 2008; Rodríguez, 2009; Sum, Mathews, Hudges \& Campbell, 2008; Tiikkainen \& Heikkinen, 2005; Tuesca, 2003) estudiaron la soledad de los adultos mayores en diferentes partes del mundo en relación con la salud, síntomas depresivos, género, aislamiento social, institucionalización, relaciones sociales, uso de nuevas tecnologías, comparación entre poblaciones, actividades sociales solitarias, factores predisponentes, entre otros.

De la misma manera, existen diferentes instrumentos para medir la soledad y la soledad social, entre ellos se encuentran las escalas UCLA, SELSA-S, ESLI, Escala de soledad, Escala de soledad social y emocional, ESTE, ESTE-R Y ESTE II.

Es clara la importancia de indagar sobre las diferentes concepciones de soledad que tienen los mayores de distintas partes del mundo, con el fin de evidenciar cómo se presenta este fenómeno en los contextos estudiados y de qué modo se relacionan o diferencian entre ellos; de esta manera, generar conocimiento de las poblaciones, sus características y realizar esfuerzos interinstitucionales de cooperación en el diseño y aplicación de estrategias de intervención adecuadas y adaptadas a cada contexto, permitiendo el mejoramiento de la calidad de vida de los adultos mayores.

Después de un amplio recorrido por las investigaciones sobre soledad realizadas en diferentes partes del mundo, se expondrán en los siguientes apartados los resultados referentes al actual estudio, es decir, la comparación de las concepciones populares de soledad de los adultos mayores de España y los adultos mayores de Bucaramanga, Colombia.

\section{Método}

\section{Propósitos de la investigación}

Comparar las concepciones populares de soledad de los adultos mayores de España y los adultos mayores de Bucaramanga, Colombia.

Analizar el concepto de soledad que tienen los mayores.

Analizar qué diferencias y semejanzas existen sobre las concepciones populares de la soledad que tiene los adultos mayores de la población española y colombiana.

\section{Diseño}

Cualitativo comparativo.

\section{Muestra}

La muestra estuvo conformada por 1956 adultos mayores de España y 6607 adultos mayores de Bucaramanga, Colombia.

Los criterios de inclusión tomados en cuenta en las 2 poblaciones fueron: ser mayor de 65 años y aceptar la participación en el estudio.

\section{Instrumentos}

A continuación se presentan los instrumentos que permitieron la comparación sobre las concepciones populares de soledad de las dos poblaciones.

1. En el estudio realizado en España se utilizó el ítem número 16 del cuestionario "La soledad en las personas mayores" cuyo contenido corresponde al concepto popular de soledad.

2. En el estudio realizado en Bucaramanga, Colombia, se utilizó la "Encuesta concepción de soledad", encuesta con única pregunta: ¿Qué es la soledad para usted? 


\section{Procedimiento}

Se retomaron los resultados tanto del ítem 16 del cuestionario "La soledad en las personas mayores" referente al concepto de soledad, como los resultados de la "Encuesta concepción de soledad", los cuales fueron utilizados en las siguientes investigaciones: Las personas mayores españolas (1999) y Concepciones populares de la soledad de los adultos mayores de Bucaramanga, Colombia (2010).

A partir de ello se realizó una comparación de las categorías arrojadas en los dos estudios.

Por último, se efectuó el análisis y discusión de la comparación por categorías frente a las poblaciones estudiadas.

\section{Resultados}

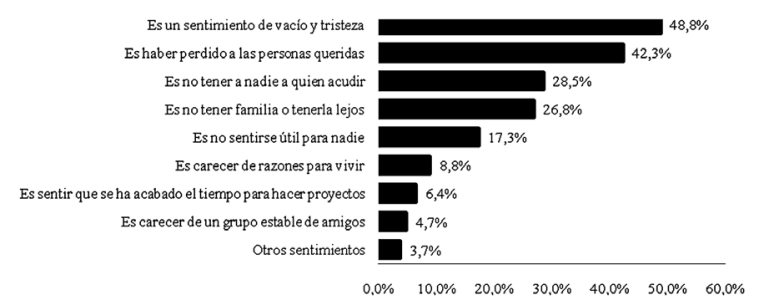

Figura 1.Concepciones populares sobre soledad de "Las personas mayores españolas".

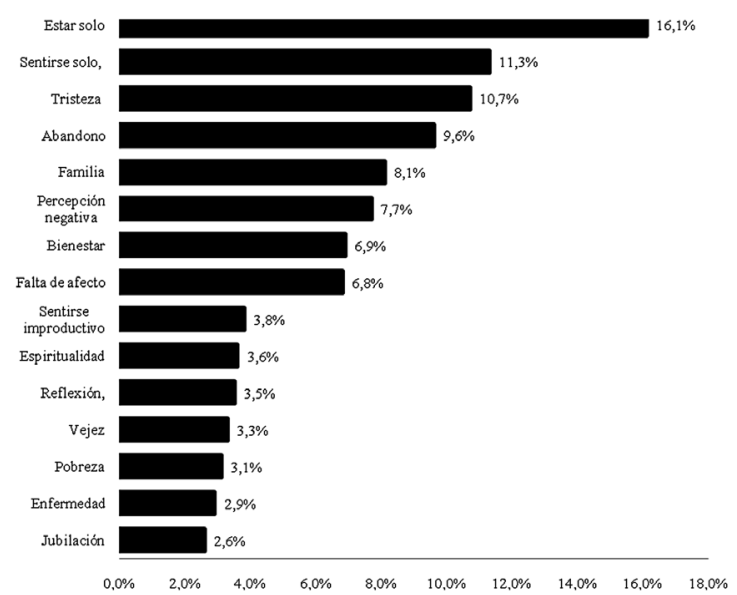

Figura 2. Concepciones populares de soledad de los adultos mayores de Bucaramanga, Colombia.
Los resultados de las concepciones populares sobre soledad arrojados en la muestra española se encuentran en la Figura 1, mientras que los resultados referentes a las concepciones populares sobre soledad de la muestra de Bucaramanga, Colombia se pueden observar en la Figura 2.

Por tanto, los porcentajes más altos arrojados en la muestra española corresponden a las categorías "Sentimiento de vacío y tristeza", "Perdida de personas queridas", mientras que en la muestra colombiana equivalen a las categorías "estar solo", "sentirse solo".

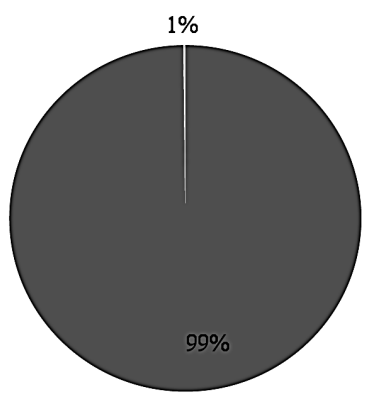

- Concepciones $\mathrm{N}$ egativas de Soledad

$\square$ Concepciones P ositivas de Soledad

Figura 3. Concepciones negativas y positivas sobre soledad de los adultos mayores de España.

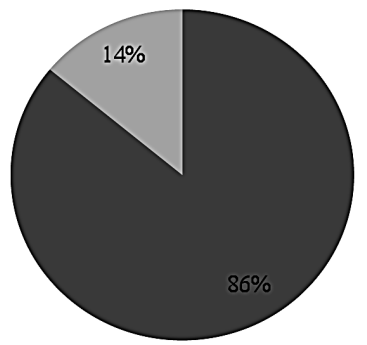

- Concepciones $\mathrm{N}$ egativas de Soledad

$\square$ Concepciones Positivas de Soledad

Figura 4. Concepciones negativas y positivas sobre soledad de los adultos mayores de Bucaramanga, Colombia.

Las figuras 3 y 4 presentan los porcentajes referentes a las concepciones populares positivas y negativas sobre soledad de los adultos mayores de España y Bucaramanga, Colombia. 


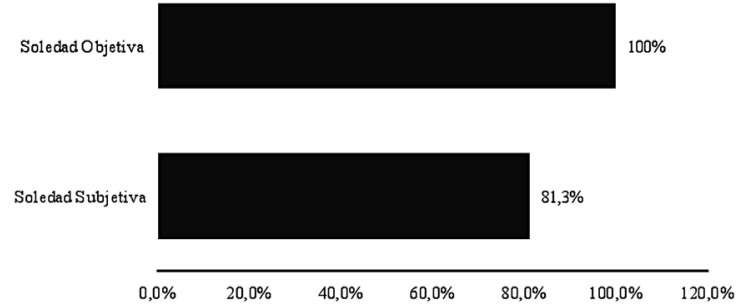

Figura 5. Concepciones populares referentes a la soledad objetiva y subjetiva de los adultos mayores de España.

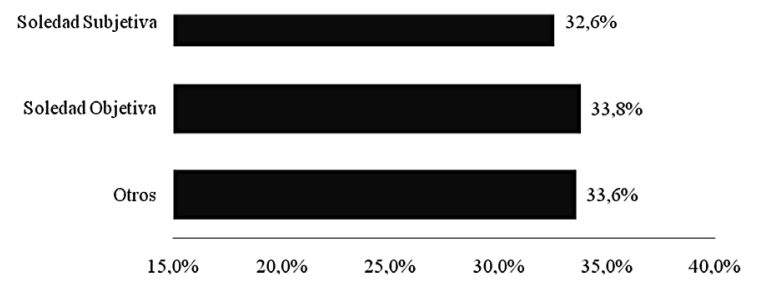

Figura 6.Concepciones populares referentes a la soledad objetiva y subjetiva de los adultos mayores de Bucaramanga, Colombia.

En las figuras 5 y 6 se presenta la sumatoria de los porcentajes de categorías que hacen referencia a la soledad subjetiva y objetiva de los adultos mayores de la población española y la población de Bucaramanga, Colombia.

\section{Discusión}

Es importante destacar que los resultados de la presente investigación revelan que tanto en la población española como en la colombiana predomina la percepción negativa de la soledad.

Baltes (1987) indica que el envejecimiento comprende un ciclo vital, que así mismo involucra una serie de ganancias y pérdidas, las cuales sin duda se presentan también en la vejez. De esta manera, la concepción negativa de la soledad se puede asociar a estos cambios, pero percibidos desde las perdidas y no desde las ganancias.

Según el Boletín sobre envejecimiento del MTAS de España (2000) la llegada a la vejez implica una serie de cambios vitales que van más allá del au- mento de años. Adaptarse a esta nueva vida no es tarea fácil y depende de los recursos personales, familiares y sociales de las personas mayores. A causa de estos cambios, pueden experimentar soledad.

Las categorías arrojadas en las dos poblaciones confirman tanto la anterior afirmación, como el estudio realizado por Iglesias (2001), pues se ven reflejadas en estos cambios vitales propios de las situaciones de carácter personal, familiar y social que se presentan en la vejez; algunos de ellos son el deterioro de facultades físicas; la viudez; la muerte de amigos y familiares de su edad; el vivir solo o la institucionalización, implicando el abandono del hogar y su rutina de vida; la pérdida de autonomía; el aislamiento social y familiar; la jubilación o el abandono laboral que conllevan al aumento del tiempo libre; entre otros.

De esta manera, los anteriores pueden considerarse elementos que llevan a que los mayores independientemente de su contexto experimenten soledad y la definan negativamente.

Existen variables que marcan diferencias en las condiciones de vida de la población española y colombiana, como por ejemplo un nivel de pobreza de la población mayor colombiana del $40 \%$, que se hace presente en las cifras encontradas por Guzman y Huenchuan en compañía del CELADE-CEPAL (2006).

Se podría suponer que estas variables se marcan como un determinante de la concepción negativa de la soledad, sin embargo, en la actual investigación esta concepción se presenta en ambas poblaciones independientemente de su situación socio-económica. Esto indica que a pesar de que las condiciones sociales pueden cambiar el estilo de vida de los mayores, el actual estudio permite suponer que estas no influyen en la concepción negativa y positiva de la soledad, y de esta manera se presenta más como una característica asociada a la vejez y no al contexto.

Otro aspecto en común que se evidencia en ambas muestras es la incidencia de categorías que implican el distanciamiento social y familiar, ha- 
ciendo énfasis en las ausencias de personas cercanas, ya sea por muerte o por abandono, y de la misma forma el aislamiento social.

Rojo (2010) investigó la calidad de vida de las personas mayores encontrando que la salud, la red familiar, las redes sociales, las actividades de ocio, se presentan como factores determinantes de la percepción de los mayores sobre su calidad de vida.

Los mayores españoles consideran que la soledad se relaciona con aspectos como "perdida de personas queridas", "no tener a nadie a quien acudir", "no tener familia o tenerla lejos"; de forma similar los mayores colombianos la asocian con el "estar solo", "sentirse solo", "abandono", "familia", "falta de afecto".

Lo anterior, muestra claramente cómo en las dos poblaciones se define la soledad en relación con las ausencias de tipo familiar y cómo la familia es considerada por el mayor número de participantes como una red de apoyo esencial para su bienestar y su calidad de vida.

Por otra parte, a pesar de que las categorías que se presentan en un mayor porcentaje son las referentes a la ausencia familiar, los mayores españoles relacionan también la soledad a "no sentirse útil para nadie", "carecer razones para vivir", "sentir que se ha acabado el tiempo para hacer proyectos", "carecer de un grupo estable de amigos", y los mayores colombianos la relacionan con "sentirse improductivo". Esto implica el aislamiento social que se presenta en las personas de edad avanzada, un distanciamiento a causa del abandono laboral, del aumento del tiempo libre, encontrándose en un mundo que está en continuo cambio y que de una manera u otra lo olvida.

Así pues, se manifiesta la importancia que tienen las redes de apoyo en las personas mayores; se puede evidenciar como los mayores relacionan la soledad con la ausencia de estas redes de apoyo, que se están viendo alteradas por los cambios propios de la vejez, de la dinámica familiar y de las nuevas formas de vida que implica la sociedad actual.
Teniendo en cuenta lo expresado en los enunciados anteriores sobre la relación entre la soledad y la ausencia familiar y social, entran a jugar factores que aclaran las características de estas ausencias, pues en ocasiones los mayores definen la soledad desde factores externos y otras veces desde factores internos como los sentimientos.

En diversos estudios (Rubio, 2004, 2009) hace una distinción sobre la soledad, mencionando que ésta ha sido vista desde dos dimensiones: la soledad objetiva "insolation" que indica el vivir solo, y la soledad subjetiva "loneliness" que supone el sentirse solo. Estos planteamientos se observan en los resultados presentados por el actual estudio, pues se sigue manifestando la separación entre la soledad objetiva y subjetiva.

Por un lado, tanto en la población española como en la colombiana se encuentran concepciones que se centran más en la soledad vinculada a la ausencia física de las personas queridas, ya sea por la muerte o la lejanía, es decir, una soledad motivada por variables externas al individuo, una soledad objetiva. Estas concepciones son: "perdida de personas queridas", "no tener a nadie a quien acudir", "no tener familia o tenerla lejos", "carecer de un grupo estable de amigos", "estar solo", "abandono", "familia".

Desde el otro punto de vista, en las dos poblaciones estudiadas se presentan concepciones de la soledad, vinculadas a las vivencias, sentimientos internos como la tristeza, que llevan a sentir y a percibir la soledad a pesar de encontrarse en un mundo lleno de personas o contar con la compañía física de estas personas, es decir, se pierde el sentido de vida y se experimenta soledad subjetiva. Estas concepciones son: "sentimiento de vacío y tristeza", "no sentirse útil para nadie", "carecer de razones para vivir", "sentir que se ha acabado el tiempo para hacer proyectos", "sentirse solo", "tristeza”, "falta de afecto", "sentirse improductivo".

Por tanto, las dos interpretaciones que dan los adultos mayores sobre la soledad (objetiva y subjetiva), presentan un denominador en común: la afectividad, así, cuando este factor se hace 
presente y domina al ser humano lleva a que los aspectos cognitivos pasen a un segundo plano, y en cualquiera de los dos casos corroborando los resultados de Rojo (2010) la persona mayor experimenta soledad, manifestada a su vez en una disminución de la calidad de vida.

En diferentes investigaciones (Cardona, 2009; Rubio \& Aleixandre, 2001) estudiaron las concepciones de la soledad de los mayores en cuanto a estar solo y sentirse solo, encontrando que a pesar de presentarse teorías implícitas de la soledad como un sentimiento subjetivo, los sujetos que viven solos generan más índices de soledad que los que viven acompañados.

Los resultados planteados en la presente comparación corroboran lo encontrado en los estudios anteriores, pues al abordar en conjunto las categorías que corresponden tanto al componente objetivo como el subjetivo de la soledad, se encuentra que en las dos poblaciones estudiadas predominan las concepciones referentes a la soledad objetiva, es decir, al estar solo, a la ausencia real de las personas.

Sin embargo, las concepciones referentes a la soledad subjetiva se manifiestan también con altos porcentajes en las dos poblaciones, porcentajes muy cercanos a los que refieren el componente objetivo de la soledad.

De forma contraria, si se analizan los porcentajes más altos arrojados en las dos poblaciones, se evidencian resultados diferentes a los planteados en el párrafo anterior, pues en la muestra española la categoría con mayor porcentaje "sentimiento de vacío y tristeza" corresponde a un sentimiento evidentemente subjetivo, seguida por la categoría "pérdida de personas queridas" es decir, soledad objetiva; mientras que en la muestra colombiana "estar solo" corresponde sin duda a la soledad objetiva, seguido por la categoría "sentirse solo" equivalente al sentimiento subjetivo.

De esta manera, la comparación de las poblaciones no manifiesta una diferencia marcada frente a la concepción objetiva y subjetiva de la soledad, sino una tendencia por separar estas dos posibi- lidades de concebirla y percibirla; independientemente de cuál prime, se debe tener en cuenta que el vivir solo es un potencial importante de la soledad, pero no exclusivo de ella, pues la soledad se presenta también en personas mayores que conviven con otras, manifestando que existe sin duda un componente subjetivo importante que debe ser considerado también como un potencial de soledad.

Otro aspecto por resaltar es la presencia de concepciones positivas como "bienestar" "espiritualidad" y "reflexión" en la soledad, en la población colombiana, a diferencia de la población española, y a pesar de que estas concepciones se manifiestan en porcentajes bajos, el hecho de que se hagan presentes es un factor importante en la investigación que indica que algunos mayores de la población colombiana conciben la soledad como un espacio productivo.

Una de las interpretaciones que se puede dar a este resultado recae en la situación socio-económica que viven los mayores colombianos a diferencia de los españoles, ya que como se muestra anteriormente, Guzmán y Huenchuan, en compañía del CELADE-CEPAL (2006) presentan datos alarmantes, como un nivel de pobreza de la población mayor colombiana del $40 \%$, esto supone que al existir un déficit en los ingresos de los mayores, algunos de ellos recurren a búsqueda de trabajos informales, poco remunerados, o que apenas ayudan a su subsistencia. Estos trabajos informales en lugar de hacer sentir productivos a los mayores, pueden estar presentándose como una sobrecarga que en muchos casos la misma familia pone sobre el mayor, de esta manera los mismos mayores pueden definir momentos de soledad como momentos de descanso y bienestar, y así mismo hacer referencia a la soledad subjetiva, pues aunque se encuentran acompañados, pueden estar percibiendo la soledad.

Para finalizar, es importante resaltar que después de hacer un recorrido por las concepciones populares sobre soledad de los adultos mayores de España y Bucaramanga, Colombia, no se llega a una definición terminada sobre soledad, si no a la aproximación de teorías implícitas de la misma, 
que de una manera u otra se manifiestan en las formas de vida, pensamientos y creencias de los adultos mayores.

\section{Conclusiones}

La soledad no tiene una única y determinada definición, ni se puede considerar rigurosamente positiva o negativa, su definición se elabora a partir de las teorías implícitas de los seres humanos que se manifiestan en su estilo de vida, creencias y pensamientos.

Los adultos mayores de las poblaciones estudiadas presentan una tendencia a definir la soledad desde una percepción negativa de la misma, asociándola con las pérdidas que se presentan en la vejez.

Las diferencias en las condiciones sociales de Colombia y España, no se muestran como un factor determinante en la concepción negativa y positiva de la soledad, presentándose más como una característica asociada a la vejez y no al contexto.

Las concepciones de soledad en las dos poblaciones tienen una estrecha relación con la ausencia directa o indirecta de la familia o personas con las que los mayores establecen vínculos afectivos, es decir, redes de apoyo.

Tanto los mayores de la población española como los de la colombiana definen la soledad desde dos perspectivas, la soledad objetiva, que hace referencia a variables externas al individuo, como el vivir solo o la ausencia física de personas, y, la soledad subjetiva, vinculada a sentimientos internos como la tristeza, que hacen que los mayores experimenten soledad en un mundo lleno de personas.

Tanto la soledad vivida como la sentida, se presentan en ambas poblaciones como un factor determinante de la soledad.

A diferencia de los mayores españoles, los mayores colombianos presentan concepciones positivas de la soledad.

Como deficiencias del estudio se encuentra la ausencia de datos sociodemográficos en la población colombiana que permitan hallar relaciones relevantes sobre las concepciones de soledad entre edad, género, religión, estrato y estado civil. De esta manera se recomienda en futuras investigaciones estudiar las concepciones populares de la soledad en la población colombiana en general, incluyendo estas características sociodemográficas; además, se sugiere seguir estudiando el fenómeno de la soledad en ambas poblaciones a través de la medición de la soledad, y la soledad social mediante la validación y aplicación de las escalas ESTE, ESTE-R, ESTE II a la población colombiana, realizando así comparaciones más detalladas.

Por último, se recomienda continuar ejecutando trabajos interinstitucionales de cooperación, con el fin de generar estrategias de intervención que contribuyan a mejorar la calidad de vida de los adultos mayores de diferentes partes del mundo.

\section{Referencias}

Alfageme, A. (1999). La soledad y el género: una aproximación factorial a la desigualdad económica, entre los ancianos españoles de los noventa. Revista multidisciplinar de gerontología, 9(3), 147-153.

Arranz, L., Giménez, L., De Castro, N. \& De la Fuente, M. (2009). El aislamiento social durante la vejez empeora el deterioro cognitivo, conductual e inmunitario. Revista española de geriatría y gerontología: órgano oficial de la Sociedad Española de Geriatría y Gerontología, 44(3), 137-142.

Baltes, P. (1987). Theoretical propositions of lifespan developmental psychology: On the dynamics between growth and decline. Developmental Psychology, 23, 611-626.

Bermejo, J. (2005). La soledad en los mayores. 13. Madrid: IMSERSO.

Bondevik, M. \& Skogstad, A. (1996). Loneliness among the oldest, old, a comparison between residents living in nursing homes and residents living in the community. International Journal of Aging y Human Development, 43(3), 181. 
Cardona, J., Henao, E., Quintero, A. \& Villamil, M. (2009). Concepto de soledad y percepción que de su momento actual tiene el adulto mayor en el municipio de Bello, Colombia, 2007. Revista Facultad Nacional de Salud Pública, 27(2), 153,163.

Cerquera, A., Muñoz, R. \& Pinzón, E. (2010). "Concepciones populares de Soledad de los adultos mayores de Bucaramanga, Colombia”. Manuscrito no publicado, Grupo de Psicología Clínica y de la Salud, Línea de Calidad de Vida en el Adulto Mayor, Universidad Pontificia Bolivariana, Bucaramanga, Colombia.

Curry, T. \& Ratliff, B. (1973). The effects of nursing home size on resident isolation and life satisfaction. The Gerontologist, 13(3), 295-298.

Departamento Administrativo Nacional de Estadísticas DANE (2010). Censo General 2005, República de Colombia, Población Adulta Mayor. Extraido el 17 de marzo de 2010 de http:// www.dane.gov.co/daneweb_V09/\#twoj_fragment1-3

Del Barrio, E., Castejón, P., Sancho, M., Tortosa, M., Sundström, G. \& Malmberg, B. (2010). La soledad de las personas mayores en España y Suecia: contexto y cultura. Revista española de geriatría y gerontología: órgano oficial de la Sociedad Española de Geriatría y Gerontología, 45(4), 189-195.

Evans, R., Werkhoven, W. \& Fox, H. (1982). Treatment of social isolation and loneliness in a sample of visually impaired elderly persons. Psychological Reports, 51(1), 103-108.

Gierveld, J. \& Van Tilburg, T. (2006). A six-item scale for overall, emotional and social loneliness: Confirmatory tests on survey data. Research on Aging: A bimonthly on Agingand the Life Course, 28, 582-598.

Guzman, J. \& Huenchuan, S. (2006). Seguridad económica y pobreza en la vejez: tensiones, expresiones y desafíos para el diseño de po- líticas. Centro Latinoamericano y Caribeño de Demografía, División de la CEPAL. Notas de población, 83. Extraído el 12 de febrero de 2011 de http://www.eclac.org/publicaciones/xml/9/30029/lcg2340-P4.pdf

Iglesias de Ussel, J. (2006). La soledad en las personas mayores: Influencias personales, familiares y sociales. Análisis cualitativo. 1-221. IMSERSO.

Instituto de Migraciones Sociales IMSERSO y Centro de Investigaciones Sociológicas CIS. (1999). La soledad en las personas mayores. Boletín 21. Extraído el 1 de febrero de 2011 de http://www.cis.es/cis/opencms/- Archivos/Boletines/21/BDO_21_index.html

Levine, S. (1980). The isolation of the community elderly from the informal social structure. Myth or reality. Journal of Geriatric Psychiatry, 13(1), 49-51.

López, J. (2004). Personas mayores viviendo solas. La autonomía como valor en alza. Madrid: IMSERSO.

Managan, D., Wood, J. \& Heinichen, C. (1974). Older adults. A community survey of health needs. Nursingresearch, 23(5), 426-432.

Ministerio de Trabajo y Asuntos Sociales. (2000). Boletín sobre el envejecimiento, La soledad de las personas mayores, 3. Madrid: IMSERSO.

Myerson, P. (1980). Intrapsychic isolation in the elderly. Journal of Geriatric Psychiatry, 13, 19-25.

Parales, C. \& Dulcey, E. (2002). La construcción social del envejecimiento y de la vejez: un análisis discursivo en prensa escrita. Revista Latinoamericana de Psicología, 34, 107-121.

Pettigrew, S. \& Roberts, M. (2008). Addressing loneliness in later life. Aging \& Mental Health, 12(3), 302-309. 
Pinazo, S. \& Sánchez, M. (2006). Gerontología, actualización, innovación y propuestas. Madrid: Prentice-Hall.

Piña, M. (2004). Gerontología social aplicada. Argentina: Editorial Espacio.

Rodríguez, M. (2009). La soledad en el anciano. Revista de la Sociedad Española de Enfermería Geriátrica y Gerontológica, 20(4), 159-166.

Rodríguez, R. (2010). Salud, amor y sexo en los adultos mayores. Guayaquil: Eduquil.

Rojo, F. (2010). Población mayor, calidad de vida y familia: demanda y prestación de cuidados en el seno familiar. Madrid: IMSERSO.

Rubio, R. \& Aleixandre, M. (1999). La escala "Este", un indicador objetivo de soledad en la tercera edad. Geriatrika. Revista Iberoamericana de Geriatría y Gerontología, 15(9), 26-35.

Rubio, R. \& Aleixandre, M. (2001). Un estudio sobre la soledad en las personas mayores: entre estar solo y sentirse solo. Revista Multidisciplinar de Gerontología, 11(1), 23-28.

Rubio, R. (2000, diciembre). Portal de Mayores. Boletín 3. La soledad en personas mayores. Soledad objetiva y soledad subjetiva.

Rubio, R. (2004). La soledad en las personas mayores españolas. Madrid: IMSERSO.

Rubio, R. (2007). La problemática de la soledad en los mayores. Infad. Revista de Psicología, 19(2).

Rubio, R. (2009). Tres interrogantes y tres respuestas sobre la soledad social en los mayores. Manuscrito no publicado. Universidad de Granada, Granada, España.
Rubio, R., Rubio, L. \& Pinel, M. (2009). La soledad en los mayores españoles: Una alternativa de solución en de la escala Este. Madrid: IMSERSO.

Rubio, R., Rubio, L. \& Pinel, M. (2009). Un instrumento de medición de soledad social, Escala Este II. Madrid: IMSERSO.

Salvarezza, L. (2002). La vejez, una mirada gerontológica actual. Buenos Aires: Paidós.

Seligman, M. (1975). Helplessness: On depression development and death. San Francisco: Freeman. Versión castellana. Madrid: Editorial Debate.

Silverstone, B. \& Miller, S. (1980). The isolation of the community elderly from the informal social structure. Myth or reality. Journal of Geriatric Psychiatry, 13(1), 27-47.

Sum, S., Mathews, M., Hudges, I. \& Campbell, A. (2008). Internet use and loneliness in older adults. Cyber Psychology y Behavior, 11(2), 208-211.

Tiikkainen, P. \& Heikkinen, R. (2005). Associations between loneliness, depressive symptoms and perceived togetherness in older people. Aging Ment Health, 9(6), 526-34.

Tuesca, R. (2003). Los grupos de socialización como factor protector contra la depresión en personas ancianas. Revista española de salud pública, 77(5), 595-604.

Zamudio, L. et ál. (2003). Colombia envejece: vámonos preparando, implicaciones e impacto del envejecimiento demográfico. Universidad Externado de Colombia. Bogotá: Santillana-AIC. 